\title{
Role of Steroid Hormone Coregulators in Health and Disease
}

\author{
M.K. Thakur V.Paramanik \\ Biochemistry and Molecular Biology Laboratory, Department of Zoology, Banaras Hindu University, Varanasi, India
}

\section{Key Words}

Steroid hormone $\cdot$ Coactivators $\cdot$ Corepressors $\cdot$ Gene regulation $\cdot$ Diseases

\begin{abstract}
The involvement of coactivators and corepressors, collectively termed as coregulators, increases the complexity of regulation of steroid hormone action. Following the interaction of the steroid hormone-receptor complex with the specific nucleotide sequences of target genes, the coregulators are recruited for activation or suppression of specific genes. The coregulators regulate a number of hormonal events during pregnancy, sex differentiation, development, reproduction and sexual behavior. They also exert equally important functions in non-reproductive tissues like heart, kidney, pancreas, bone and brain. The mutation and/or aberrant expression of these coregulators affect the normal function of steroid hormones and result in physiological abnormalities leading to the development of diseases. Therefore, understanding the role of coregulators in steroid hormone action is important and would help in developing the therapeutic strategy for the treatment of steroid-related diseases. In this review article, we describe the coregulators and their implication in health and pathogenesis of diseases. Furthermore, the possible therapeutic approach has been discussed for the treatment of steroid-related diseases, which will be of future interest in the field of medical sciences.
\end{abstract}

Copyright $\odot 2009$ S. Karger AG, Basel

\section{Introduction}

Steroid hormone action is mediated mainly through the intracellular nuclear receptors. These receptors are ligand-inducible transcription factors belonging to the nuclear receptor superfamily and mediate diverse actions of sex steroids. They share three common functional domains: (i) amino-terminal transactivation domain containing activation function (AF)-1, (ii) central or DNAbinding domain (DBD) containing two zinc fingers like motifs, and (iii) carboxy-terminal ligand-binding domain containing AF-2 [1]. Following the binding of hormone to its specific receptor, the binary complex interacts with the

ACTR = Activator of the thyroid and retinoic acid receptor; ARA = androgen receptor activator; ARIP = androgen receptor-interacting protein; $B R G$ = Brahma-related gene; $C B P=C R E B$-binding protein; $\mathrm{CiZ1}=\mathrm{Cip}$-interacting zinc finger protein $1 ; \mathrm{CREB}=\mathrm{CAMP}$-response element-binding protein; DAX-1 = DSS-AHC critical region on the chromosome gene 1; DRIP = vitamin D receptor-interacting protein; ERAP-140 = estrogen receptor (ER)-associated protein 140; ETO = eight-twenty-one translocation; GRIP = glucocorticoid receptor interacting protein; $\mathrm{MeCP}-2$ = methyl-CpG-binding protein; $\mathrm{NCOR}=$ nuclear receptor corepressor; $\mathrm{pCAF}=\mathrm{p} 300 / \mathrm{CBP}$-associated factor; $\mathrm{pCIP}=\mathrm{p} 300 / \mathrm{CBP}$ cointegrator-associated protein; $\mathrm{PGC}=\mathrm{PPAR} \gamma \mathrm{Co}-$ activator; RAC = receptor-associated coactivator; RIP = receptor-interacting protein; SMRT = silencing mediator of retinoic acid and thyroid hormone receptor; $\mathrm{SRC}=$ steroid receptor coactivator; $\mathrm{TAF}=$ TBP-associated factor; TBP = TATA-binding protein; TIF = transcriptional intermediary factor.

\section{KARGER}

Fax +4161306 1234 E-Mail karger@karger.ch www.karger.com
(C) 2009 S. Karger AG, Basel

0301-0163/09/0714-0194\$26.00/0

Accessible online at:

www.karger.com/hre
M.K. Thakur

Biochemistry and Molecular Biology Laboratory

Department of Zoology, Banaras Hindu University

Varanasi-221 005 (India)

Tel. +91 542231 3958, Fax +91 542236 8174, E-Mail mkt_bhu@yahoo.com 
Fig. 1. Role of coregulators in gene regulation during steroid hormone action.

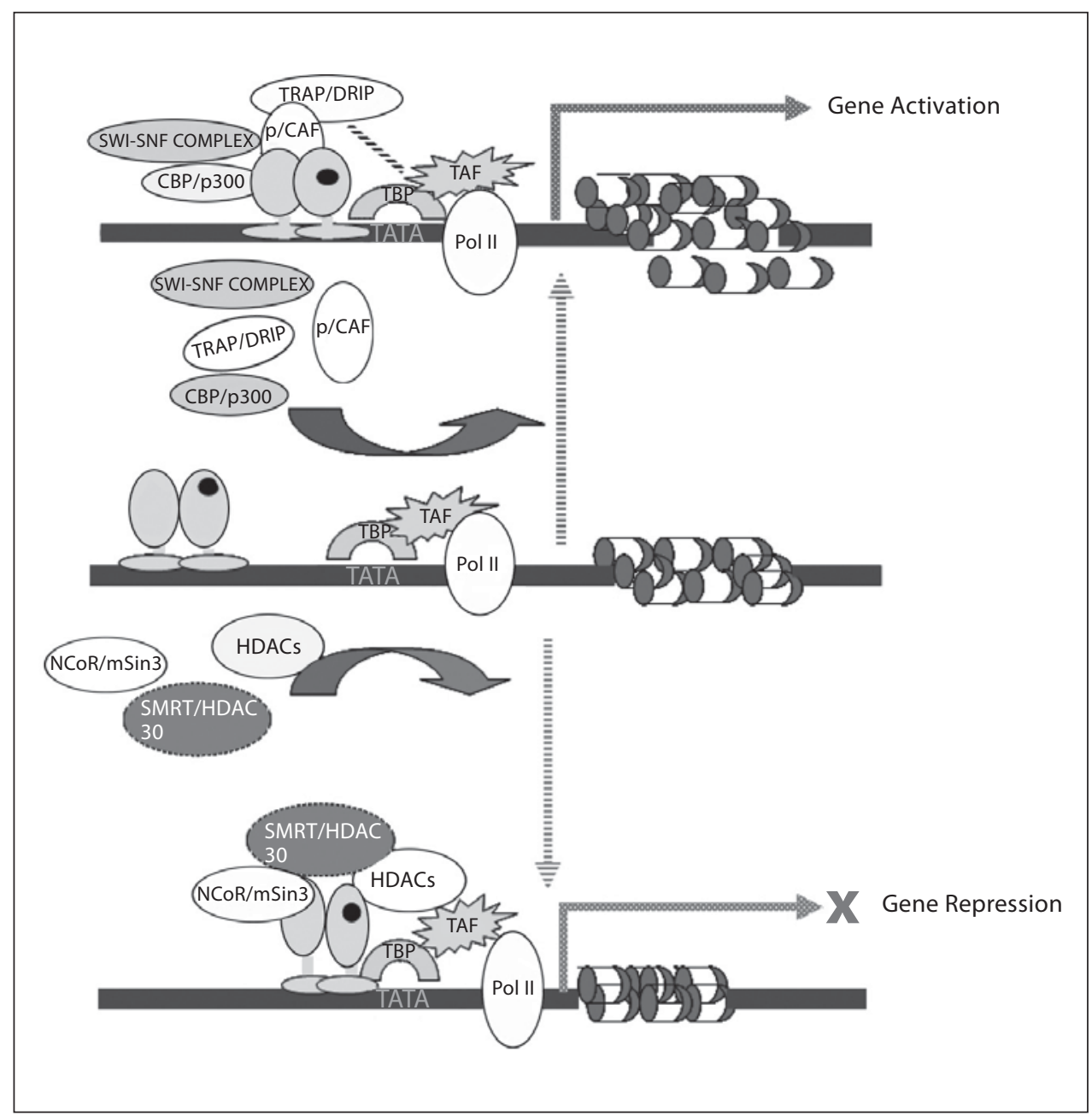

responsive elements of the target genes and recruits an array of coregulators and chromatin modifiers. The involvement of coregulators makes the regulation more complex (fig. 1). These coregulators are classified as coactivators when they activate the expression of genes and as corepressors when they inhibit the transcription of genes [2]. Previously, it was thought that coactivators and corepressors are present in separate complexes, but recent evidence indicates that these molecules exist in the same complex [3]. This complex has an opposite function and allows for efficient transcriptional control mechanisms depending on posttranslational modification of coregulators. In the majority of cases, transcription is activated by histone acetylation and repressed by histone deacetylation.

This review discusses the current understanding of coregulators and their mechanism of action, defects in coregulators, resulting diseases and therapeutic ap- proaches. The information included in this review has been collected from the literature available in NCBI, ISI Web of Knowledge, ScienceDirect search engine, HighWire press and Karger press journals. Understanding the role of coregulators in steroid hormone action is important for developing a therapeutic strategy for the treatment of steroid-related diseases.

\section{Coactivators and Their Mechanism of Action}

A large number of coactivators have been identified and characterized over the past few years [4] - several are listed in table 1 . They are characterized by their interaction with the transcription machinery and activation function domains of nuclear receptors [5]. Based on the mechanism of function, coactivators can be categorized 
Table 1. Coactivators and their interaction with nuclear receptors

\begin{tabular}{|c|c|c|}
\hline \multirow[t]{2}{*}{ Coactivators } & \multicolumn{2}{|l|}{ Interaction } \\
\hline & in vitro & in vivo \\
\hline SRC & PR, RAR, RXR, TR & ER, GR, PR, TR, RXR \\
\hline ERAP160/P 160 & ER, RAR, RXR & ER \\
\hline GRIP1/TIF2 & ER, AR, GR, TR, PR, RAR, RXR & $\mathrm{ER}, \mathrm{AR}, \mathrm{GR}, \mathrm{PR}$ \\
\hline SRC-2/NcoA-2 & PPAR & VDR, RAR, RXR \\
\hline ACTR/AIB1/RAC3 & ER, PR, TR, VDR, RAR, RXR, VDR, PPAR & ER, PR, TR, RAR \\
\hline $\mathrm{p} / \mathrm{CIP}$ & ER, RAR & ER, PR, TR, RAR \\
\hline ERAP140/P140 & ER & not available \\
\hline RIP140 & ER, PPAR $\alpha, T R, R A R, R X R$ & ER \\
\hline RIP160/P160 & ER & not available \\
\hline $\mathrm{p} / \mathrm{CAF}$ & ER, AR, GR, RAR, RXR & RAR/RXR \\
\hline $\mathrm{CBP} / \mathrm{P} 300$ & ER, GR, TR, RAR, RXR & ER, TR, RAR,RXR \\
\hline ARA70 & AR & AR, ER, GR, PR (weak) \\
\hline Ada3 & ER, TR, RXR & ER, RXR \\
\hline Rap46 & ER, AR, GR, AR, PR, TR & not available \\
\hline GRIP170 & GR & GR \\
\hline PGC-1 & $\mathrm{ER} \alpha, \operatorname{PPAR} \gamma, \operatorname{RAR} \alpha, \mathrm{TR} \beta$ & $\begin{array}{l}\operatorname{PPAR} \gamma / \mathrm{RAR} \alpha, \mathrm{TR} \beta / \\
\operatorname{RXR} \alpha\end{array}$ \\
\hline PGC-2 & $\operatorname{PPAR} \gamma, \mathrm{ER} \alpha, \mathrm{TR} \beta$ & $\operatorname{PPAR} \gamma, \mathrm{ER} \alpha$ \\
\hline SPT6 & ER & ER \\
\hline SW12/SNF2 (Brahma) & GR (SWI3), ER & ER, GR, RAR \\
\hline SNURF & with $\mathrm{DBD}$ of $\mathrm{AR}, \mathrm{ER}, \mathrm{PR}$ & AR \\
\hline TRAP220 & TR, VDR, RAR, RAR,RXR, PPAR $\alpha$, PPAR $\gamma$, ER & $\mathrm{TR}$ \\
\hline TRAP100 & $\begin{array}{l}\text { ER, RXR, PPAR } \alpha, \text { PPAR } \gamma, \text { RAR and RXR } \\
\text { associate with a different complex }\end{array}$ & VDR \\
\hline DRIP & TR, VDR, PPAR $\gamma$ & VDR \\
\hline RAC3 & $\mathrm{AR}, \mathrm{ER}$ & AR, ER \\
\hline NSD & ER, RXR & Bifunctional factor \\
\hline & TR, RAR & Repression and activation \\
\hline RSP5/RPF1 & no direct interaction & GR, PR, but not ER \\
\hline PGC-I & PPAR, liver X receptor & PPAR, liver X receptor \\
\hline ARI4 & AR & AR \\
\hline CiZ/FHL2 & $\mathrm{ER} \alpha$ & $\mathrm{ER} \alpha$ \\
\hline Ubc9/PIAs & $\mathrm{ER} \alpha$ & $\mathrm{ER} \alpha$ \\
\hline
\end{tabular}

into three distinct multiprotein complexes: (i) SWI/SNF complex, which is associated with ATP-dependent alteration in chromatin structure; (ii) $p 160 / C B P$ complex, which is primarily related to histone acetylation, and (iii) mediator complex, which is involved in the activation of RNA polymerase II and initiation of transcription.

\section{(i) SWI/SNF Complex and Chromatin Remodeling}

Members of the SWI/SNF family of coactivators are the first described positive regulators of hormone action. They were initially identified in the yeast on the basis of a genetic screening required for regulation of mating type switching [6]. They mediate chromatin remodeling of swi/ snf genes and support glucocorticoid receptor (GR)-de- pendent transactivation. However, mutations in these genes fail to transactivate a reporter gene in the presence of transfected GR. It has also been shown that GR co-immunoprecipitates with the SWI/SNF complex $[7,8]$. Ligand-dependent transcriptional activation of GR or estrogen receptor (ER) requires several SWI gene products such as SWI1, SWI2 and SWI3, which are members of the SWI/ SNF complex. The human homologue of SWI2 is termed as SNF $2 \alpha$ and SNF2 $\beta$ or Brahma $[9,10]$. Other members of the SW1/SNF family include the yeast general control non-repressed protein (GCN) 5, p300 and CBP [11, 12]. They possess histone acetyltransferase (HAT) activity which is involved in chromatin remodeling. 
Table 2. Corepressors and their interaction with nuclear receptors

\begin{tabular}{lll}
\hline \multirow{2}{*}{ Corepressors } & Interaction & \\
\cline { 2 - 3 } & in vitro & in vivo \\
\hline SMRT/TRAC-2 & RAR, TR, v-ErbA, PPAR $\gamma$, & RAR, TR, PPAR $\gamma$ \\
& RXR (weak) & \\
N-CoR/RIP13 & TR, Rev Erb, RAR, PPAR $\gamma$ & TR, Rev Erb, RAR \\
SUN-CoR & TR, Rev Erb & TR, Rev Erb \\
Ssn6/Tup1 & Not available & ER, PR \\
TRUP & TR & TR, RAR \\
Calreticulin & GR, AR & GR, AR, RAR \\
REA & ER & ER \\
ZNF 366 & ER $\alpha$ & ER \\
RIP 140 & AR, ER $\alpha$ & AR, ER $\alpha$ \\
MTA & ER $\alpha$ & ER $\alpha$ \\
NM23-H2 & ER $\beta$ & ER $\beta$ \\
AIB & ER $\alpha$ & ER $\alpha$ \\
\hline
\end{tabular}

(ii) $p 160 / C B P$ and Histone Acetylation

SRC-1, first discovered in human as a ligand-dependent protein of progesterone receptor (PR), belongs to the p160/CBP complex [13]. It interacts with helix 12 within AF-1 and AF-2 regions of ER [14]. The binding of SRC-1 is more efficient when it makes a bridge between AF-1 and AF-2 and interacts directly to the basal transcription machinery through TFII or TBP [15].

DRIP, purified previously as a member of the p160/ CBP coactivator complex, has no HAT activity [16]. VDR interacts directly with a human multi-protein WINAC through the William syndrome transcription factor which contains both SWI/SNF components and DNA replication-related factors and exhibits ATP-dependent chromatin remodeling activity [17]. Another member of the p160/CBP complex, p300/CBP, interacts with roughly 100 proteins [18]. RAC3, also known as SRC-3/ACTR/ AIB1/pCIP/TRAM-1, acts through a bipartite nuclear localization signal and importin $\alpha 3$ protein [19]. AIB (amplified in breast cancer) 1 , a member of the p160 family, is highly expressed in ER- and PR-responsive endometrial carcinoma [20].

\section{(iii) Chromatin Modifiers and RNA Polymerase II}

Higher order chromatin structure is regulated in part by covalent modifications of $\mathrm{N}$-terminal histone tails, which in turn serve as platforms for the recruitment of signaling molecules that include non-histone proteins such as heterochromatin protein (HP1), nucleosomal re- modeling and histone deacetylation complex [21]. Translocated in liposarcoma (TLS), a $65-\mathrm{kDa}$ protein has been identified as TFII/RNA polymerase II-associated protein because of its sequence similarity to hTAF68 [22]. Its high-affinity binding enhances the expression of thyroid receptor (TR) proteins. Furthermore, sumoylation is responsible for chromatin modification of AR interacting protein 4 (ARIP4), which belongs to the SNF2-like family and acts as a chromatin modifier in AR regulation. However, the ability of ARIP4 to activate AR function is abolished with mutation in six potential SUMO (small ubiquitin-related modifier) attachment sites [23]. Other chromatin modifications may take place by sumoylation or binding of cofactors. For example, the chromatin remodeling factor Mi- $2 \alpha$ interacts with human c-Myb and activates the transcription of hematopoietic genes [24].

\section{Corepressors and Their Mechanism of Action}

As compared to coactivators, relatively less is known about corepressors (table 2). A true corepressor contains autonomous repression domain and directly interacts with the receptor. It also interacts with components of the transcription machinery and represses the transcription of a specific gene. The repression of transcription may be due to competition among molecules which bind to DNA or may be due to direct silencing of the basal transcription machinery [25]. Thus, a particular gene may be repressed through the recruitment of corepressor molecules to the gene and protein-protein interaction. TR-mediated repression is reversed by cotransfection with either the unliganded retinoic acid receptor (RAR) or C-terminus of the oncoprotein (v-ErbA) [26].

The corepressors are associated with nuclear hormone receptors and recruit histone deacetylase (HDAC) 3 and other isoforms that mediate histone deacetylation [27]. Another interacting protein, ZNF366, acts directly or through ER $\alpha$ corepressors, namely RIP140 and CtBP, and inhibits the expression of estrogen-responsive genes by HDAC activity [28]. These findings strongly support the hypothesis that gene transcription is repressed through nucleosomal condensation caused by deacetylation of histones [29].

Both corepressors NCoR and SMRT exist in large protein complexes with estimated sizes of 1.5-2 $\mathrm{MDa}$ in HeLa nuclear extracts. Using conventional and immunoaffinity chromatography, HDAC3, transducin ( $\beta$ )-like 1 and a WD-40 repeat-containing protein have been isolated as subunits of the purified SMRT complex. The 
HDAC3-containing SMRT and NCoR complexes can bind to unliganded thyroid hormone receptors (TRs) in vitro. The injection of antibodies against HDAC3 or SMRT/NCoR into Xenopus oocytes leads to partial relief of repression by unliganded TR/RXR, suggesting both NCoR and SMRT as partner of HDAC-containing complexes and their function in transcriptional repression [30].

\section{Coregulators and Diseases}

Steroid receptors in combination with their coactivators and corepressors mediate precise gene regulation ranging from the ligand-induced transcriptional activation to repression. As studies continue to unfold the role of coregulators in transcriptional regulation by nuclear steroid receptors, it is becoming evident that they are important for understanding the etiology of a variety of diseases such as obesity, diabetes, atherosclerosis, osteoporosis, cancer, neurodegenerative disorders, etc. The coregulators may also be used as therapeutic targets in steroid-related diseases. For example, breast cancer patients show resistance to anticancer drugs, and the majority of them eventually do not respond to tamoxifen therapy [31].

\section{Coactivators and Diseases}

Mutations in CBP coactivator are involved in embryogenesis and chromosomal translocation of $\mathrm{p} 300$ gene that causes Rubinstein-Taybi syndrome. The loss of one functional copy of the CBP gene leads to the developmental abnormalities in Rubinstein-Taybi syndrome and possibly the propensity for malignancy [32]. It also causes open neural tube and heart defects in humans, as well as embryonic lethality in mice $[33,34]$. The activity of CBP/ p300 is also inhibited in neurodegenerative diseases [35].

Coactivators like Ciz1 and FHL2 (four and half LIM domain) interact with $\mathrm{ER} \alpha$ and are involved in ovarian and breast cancers $[36,37]$. The peroxisome proliferatoractivated receptor (PPAR) $\gamma$ coactivator-1 of the PGC-1 gene family regulates lipid and carbohydrate metabolism and inflammation [38]. Knockout studies reveal the effects of two members of the p160 family on energy homeostasis. TIF2-/- mice are protected against obesity and display enhanced adaptive thermogenesis, whereas SRC1-/- mice are prone to obesity due to reduced energy expenditure [39]. In addition, TRAP220 knockout re- veals an early gestational stage with heart failure and exhibits impaired neuronal development with extensive apoptosis [40].

Mutations in receptor gene also inhibit the binding of coactivators and lead to diseases. For example, androgen-insensitive syndrome is developed due to mutation in the DNA-binding domain of AR which prevents interaction of coactivators to the AF-1 region and transmission of activation signal [41]. This syndrome shows various degrees of impairment in the genital virilization $[42,43]$. The transcriptional response to AR coactivator (ART-27) is increased by the majority of naturally occurring AR mutations in prostate cancer, but decreased by P340L mutation and androgen-insensitive syndrome [44].

\section{Corepressors and Diseases}

Mutation in the corepressor or change in its interaction with the basal transcription machinery may lead to diseases. For example, mutations in MeCP-2, RB and DAX-1 result in the loss of corepressor interaction leading to embryonic diseases during development $[45,46]$. Mutation in MeCP-2, which affects either the methylbinding domain or the transrepressor domain by deacetylation, causes an X-linked neurodegenerative developmental disorder, Rett syndrome [47]. These mutations result in the loss of recruitment of the SIN3 corepressor to target genes [48]. Mutations in MTA (metastasis-associated protein) 2 are responsible for estrogen-responsive cancer [49]. An ER $\beta$-associated corepressor NM23$\mathrm{H} 2$ has been identified in the arteries from young subjects (27 \pm 6 years) with benign intimal hyperplasia. The expression of NM23-H2 decreases with the advancement of atherosclerosis and inflammation in human [50].

Mutations in receptors also affect their interaction with corepressors and lead to a number of diseases. For example, acute myelogenic leukemia (AML), which results from translocations involving fusion of $\mathrm{DBD}$ of AML protein to the transcriptional repressor $\mathrm{ETO} /$ MTG8, is associated with aberrant interaction of corepressor with transcription factors [51]. Similarly, B-cell acute lymphoblastic leukemia involves the chromosomal translocation, which results in the fusion of repression domain of the Ets leukemia protein TEL to the large form of AML. In both cases, corepression arises from the recruitment of NCoR and SIN3-HDAC complex by ETO and TEL [52]. 


\section{Therapeutic Approach}

The synthetic compounds, which target HAT/HDAC complex or the receptor itself, are used in the therapy of diseases caused by defects in the binding of coregulators.

\section{Targeting HAT/HDAC Complex}

The reduction in HDAC activity and HDAC2 expression causes chronic obstructive pulmonary disease which may account for the amplified inflammation and resistance to the actions of corticosteroids. The reduction in HDAC2 expression may be secondary to the oxidative and nitrative stress as a result of cigarette smoking and severe inflammation. It occurs to different degrees in severe asthma, smoking asthmatic patients and cystic fibrosis. Similar mechanisms may also account for the steroid resistance seen in latent adenovirus infections. The reduction in HDAC activity induced by oxidative stress can be restored by theophylline, which acts through specific kinases and reverses steroid resistance in chronic obstructive pulmonary disease and other inflammatory lung diseases [53]. Several histone deacetylase inhibitors have been shown to inhibit tumor growth and now used in clinical trials for the treatment of cancer $[54,55]$. The inhibition of HDAC1 by phenylbutyrate also reverses the ETO-mediated transcriptional repression and induces partial differentiation of AML-ETO cell line [56].

\section{Targeting Receptors and Binding Partners}

LXRs (LXR $\alpha$ and LXR $\beta$ ) have a sterol-responsive property and can bind directly with several oxysterol metabolites. They are involved in cholesterol transport, glucose metabolism and inflammation. Thus, synthetic LXR ligands have been designed to treat disorders such as atherosclerosis and diabetes [57]. Further, statins modulate the sterol-responsive element-binding protein, which targets genes that are involved in cholesterol and fatty acid metabolism, namely hydroxyl-methyl-glutaryl acetyl Coenzyme-A (HMG-CoA) reductase, HMG-CoA synthase and the low-density lipoprotein receptor [58]. Similarly, insulin-like drugs target the PPAR $\gamma$, whereas several anti-inflammatory drugs inhibit activation of nuclear factor- $\kappa \mathrm{B}[59]$.

\section{Conclusion}

The expanding number of coregulators has increased the challenge and added the complexity to understanding the transcriptional regulation of steroid hormone action. One of the future challenges is to determine the specificity of coregulators. For example, the central nervous system is regulated by an array of NR coactivators such as p160/SRC family members, CBP/p300, BRG1, TRAP220 and PGC- $1 \alpha$ as well as corepressors like NCoR and SMRT. In recent years, the study of corepressor biology has become an area of active research. This has led to substantial progress in the identification of a number of novel corepressors with critical roles in determining the action of NR. Emerging data re-emphasize that the regulation of NR by corepressor is as significant as that by coactivator. Although much more work remains to be done, we have begun to gain a deeper insight into the transcriptional regulation of steroid receptors, the role played by chromatin modifications, and the contribution of coactivators and corepressors in the development of pathological conditions. Further study of key coregulators in physiologically relevant animal models and human subjects combined with cell culture models will help to elucidate the detailed mechanism of steroid hormone action and develop the therapeutic strategies for the hormone-related diseases.

\section{Acknowledgements}

We acknowledge the financial support from the Indian Council of Medical Research and Department of Biotechnology, Government of India.

\section{References}

Evans RM, et al: The steroid and thyroid hormone receptor superfamily. Science 1988 ; 240:889-895.

2 Kumar R, et al: The clinical relevance of steroid hormone receptor corepressors. Clin Cancer Res 2005;8:2822-2830.

3 Helmut D, et al: Agonist-antagonist induced coactivator and corepressor interplay on the human androgen receptor. Mol Cell Endocrinol 2003;213:79-85.

\footnotetext{
4 McKenna NJ, Malley BWO: Combinatorial control of gene expression by nuclear receptor and coregulators. Cell 2002; 108:465474.

5 Hong $\mathrm{H}$, et al: GRIP1, a novel mouse protein that serves as a transcriptional coactivator in yeast for the hormone-binding domains of the steroid receptors. Proc Natl Acad Sci USA 1996;93:4948-4952.
} 
6 Breeden L, Nasmyth K: Similarity between cell cycle genes of budding yeast and fission yeast and the notch gene of Drosophila. Nature 1987;329:651-654.

-7 Yoshinaga SK, et al: Roles of SWI1 SWI2, and SWI3 proteins for translational enhancement by steroid receptors. Science 1992;258: 1598-1604.

$\checkmark 8$ Kwon H, et al: Nucleosome disruption and enhancement of activator binding by a human SW1/SNF complex. Nature 1994;370: 477-481.

$\checkmark 9$ Chiba H, et al: Two human homologues of Saccharomyces cerevisiae SWI2/SNF2 and Drosophila Brahma are transcriptional coactivators cooperating with the estrogen receptor and the retinoic acid receptor. Nucl Acid Res 1994;10:1815-1820.

10 Peterson CL, et al: Five SWI/SNF gene products are components of a large multisubunit complex required for transcriptional enhancement. Proc Natl Acad Sci USA 1994;8: 2905-2908.

$\checkmark 11$ Candau R, et al: Histone acetyltransferase activity and interaction with ADA2 are critical for GCN5 function in vivo. EMBO J 1997; 16:555-565.

12 Ogryzko VV, et al: The transcriptional coactivators $\mathrm{p}^{300}$ and CBP are histone acetyltransferase. Cell 1996;87:953-959.

13 Onate SA, et al: Sequence and characterization of a coactivator for the steroid receptor superfamily. Science 1995;270:1354-1357.

14 Kalkhoven E, et al: Isoforms of steroid receptor coactivator 1 differ in their ability to potentiate transcription by the oestrogen receptor. EMBO J 1998;17:232-243.

$\checkmark 15$ McInerney EM, et al: Analysis of estrogen receptor transcriptional enhancement by a nuclear hormone receptor. Proc Natl Acad Sci USA 1996;93:10069-10073.

-16 Rachez C, Freedman LP: Mechanisms of gene regulation by vitamin $\mathrm{D}_{3}$ receptor: a network of coactivator interactions. Gene 2000;246:9-21.

17 Hirochika K, et al: The chromatin remodeling complex WINAC targets a nuclear receptor to promoters and is impaired in Williams syndrome. Cell 2003;113:905-917.

18 Janknecht R: The versatile functions of the transcriptional coactivators p300 and CBP and their roles in disease. Histol Histopathol 2002;17:657-668.

19 Yeung PL, et al: Nuclear localization of coactivator RAC3 is mediated by a bipartite NLS and importin $\alpha 3$. Biochem Biophys Res Commun 2006;1:13-24.

20 Balmer NN, et al: Steroid receptor coactivator AIB1 in endometrial carcinoma, hyperplasia and normal endometrium: correlation with clinicopathologic parameters and biomarkers. Mod Pathol 2006;12:1593-1605.

-21 Leader JE, et al: Epigenetics and the estrogen receptor. Ann NY Acad Sci 2006;1089:7387.

22 Bertolotti A, et al: hTAF(II)68, a novel RNA/ ssDNA-binding protein with homology to the pro-oncoproteins TLS/FUS and EWS is associated with both TFIID and RNA polymerase II. EMBO J 1996;18:5022-5031.
23 Domanskyi A, et al: Biochemical characterization of androgen receptor-interacting protein 4. Biochem J 2006;3:789-795

24 Saether T, et al: The chromatin remodeling factor $\mathrm{Mi}-2 \alpha$ acts as a novel co-activator for human c-Myb. J Biol Chem 2007;282:1399414005.

25 Horwitz KB, et al: Nuclear receptor coactivators and corepressors. Mol Endocrinol 1996, 10:1167-1177.

26 Baniahmad A, et al: A transferable silencing domain is present in the thyroid hormone receptor, in the v-erbA oncogene product and in the retinoic acid receptor. EMBO J 1992; 11:1015-1023.

27 Takahiro I, Lazar MA: The NCoR/histone deacetylase 3 complex is required for repression by thyroid hormone receptor. Mol Cell Biol 2003;23:5122-5131.

28 Lopez-Garcia J, et al: ZNF366 is an estrogen receptor corepressor that acts through CtBP and histone deacetylases. Nucleic Acid Res 2006;21:6126-6136.

29 Wolffe AP, Pruss D: Targeting chromatin disruption: transcription regulators that acetylate histones. Cell 1996;84:817-819.

30 Li J, et al: Both corepressor proteins SMRT and $\mathrm{NCoR}$ exist in large protein complexes containing HDAC3. EMBO J 2000;19:43424350.

31 Wang LH, et al: Disruption of estrogen receptor DNA-binding domain and related intramolecular communication restores tamoxifen sensitivity in resistant breast cancer. Cancer Cell 2006;6:4874-4899.

32 Fred P, et al: Rubinstein-Taybi syndrome caused by mutations in the transcriptional coactivator CBP. Nature 2002;376:348-351.

33 Yao TP, et al: Gene dosage-dependent embryronic development and proliferation defects in mice lacking the transcriptional integrator p300. Cell 1998;93:361-372.

34 Kung AL, et al: Gene dose-dependent control of hematopoiesis and hematologic tumor suppression by CBP. Gen Dev 2000;14:272-277.

35 Neri C: New light on polyglutamine neurodegenerative disorders: inference with transcription. Trend Mol Med 2001;7:283-284.

36 Kobayashi S, et al: FHL2, UBC9 and PIAS1 are novel estrogen receptor $\alpha$-interacting proteins. Endocr Res 2004;4:617-621.

37 Den Hollander P, et al: Ciz1, a novel DNA binding coactivator of the estrogen receptor $\alpha$ confers hypersensitivity to estrogen action. Cancer Res 2006;22:11021-11029.

38 Glass CK: Going nuclear in metabolic and cardiovascular disease. J Clin Invest 2006;3: $556-560$.

39 Frederic P, et al: SRC-1 and TIF2 control energy balance white and brown adipose tissues. Cell 2002;111:931-941.

40 Ito M, et al: Involvement of the TRAP220 component of the TRAP/SMCC coactivator complex in embryonic development and thyroid hormone action. Mol Cell 2000;5:683693.

41 Imasaki K, et al: Androgen insensitivity syndrome due to new mutations in the DNAbinding domain of the androgen receptor. Mol Cell Endocrinol 1996;120:15-24. $\checkmark 42$ Eli YA, Jon DH: Single-gene mutations resulting in reproductive dysfunction in women. N Engl J Med 1999;340:709-718.

43 Zoppi S, et al: Complete testicular feminization caused by amino-terminal truncation of the androgen receptor with downstream initiation. J Clin Invest 1993;91:1105-1112.

$44 \mathrm{Li} \mathrm{W}$, et al: Androgen receptor mutations identified in prostate cancer and androgen insensitivity syndrome display aberrant ART-27 coactivator function. Mol Endocrinol 2005;9:2273-2282.

45 Nan X, et al: Transcriptional repressions by methyl-CpG-binding protein $\mathrm{MeCP} 2$ involves a histone deacetylase complex. Nature 1998;393:386-389.

46 Batsche E, et al: Rb enhances p160/SRC coactivator-dependent activity of nuclear receptors and hormone responsiveness. J Biol Chem 2005;280:19746-19756.

47 Kouzarides T: Histone acetylases and deacetylases in cell proliferation. Curr Opin Gen Dev 1999;9:140-148.

48 Wong CW, Privalsky ML: Transcriptional repression by the SMRT-mSin 3 corepressor: multiple interactions, multiple mechanisms, and a potential role for TFIIB. Mol Cell Biol 1998; 18:5500-5510.

49 Cui Y, et al: Metastasis-associated protein 2 is a repressor of estrogen receptor $\alpha$ whose overexpression leads to estrogen-independent growth of human breast cancer cells. Mol Endocrinol 2006;9:2020-2035.

50 Rayner K, et al: NM23-H2, an estrogen receptor $\beta$-associated protein, shows diminished expression with progression of atherosclerosis. Am J Physiol 2007;292:R743-R750.

51 Fenrick R, et al: Both TEL and AML-1 contribute repression domains to the $\mathrm{t}(12 ; 21)$ fusion protein. Mol Cell Biol 1999;10:6566-6574.

52 Lutterbach B, et al: A target of $\mathrm{t}(8 ; 21)$ in acute leukemia interacts with the NCoR and $\mathrm{mSin} 3$ corepressors. Mol Cell Biol 1988;18:71767184.

53 Adcock IM, et al: Histone deacetylation: an important mechanism in inflammatorylung diseases. J Chron Obstruct Pulmon Dis 2005; 4:445-455.

54 Secrist JP, et al: HDAC inhibitors for the treatment of cancer. Curr Opin Invest Drugs 2003;4:1422-1427.

55 Bicaku E, et al: Selective inhibition of histone deacetylase 2 silences progesterone receptormediated signaling. Cancer Res 2008;68: 1513-1519.

56 Wang J, et al: Inhibitors of histone deacetylase relieve ETO-mediated repression and induce differentiation of AML1-ETO leukemia cells. Cancer Res 1999;12:2766-2769.

57 Michael LF, et al: The pharmacology of LXR. Med Chem 2005;8:729-740.

58 De Nigris F, et al: Coregulators new insights in the transcriptional activity and coregulator molecules in the arterial wall. Int J Cardiol 2002;2-3:153-168

59 Tanaka T, et al: Ligands for peroxisome proliferator-activated receptors and inhibit chemically induced colitis and formation of aberrant crypt foci in rats. Cancer Res 2001; 61:2424-2428. 